\title{
The Function of Words: Distinct Neural Correlates for Words Denoting Differently Manipulable Objects
}

\author{
Shirley-Ann Rueschemeyer ${ }^{1}$, Daan van Rooij ${ }^{1}$, Oliver Lindemann ${ }^{1}$, \\ Roel M. Willems ${ }^{2}$, and Harold Bekkering ${ }^{1,2}$
}

\begin{abstract}
Recent research indicates that language processing relies on brain areas dedicated to perception and action. For example, processing words denoting manipulable objects has been shown to activate a fronto-parietal network involved in actual tool use. This is suggested to reflect the knowledge the subject has about how objects are moved and used. However, information about how to use an object may be much more central to the conceptual representation of an object than information about how to move an object. Therefore, there may be much more fine-grained distinctions between objects on the neural level, especially related to the usability of manipulable objects. In the current study, we investi-
\end{abstract}

\section{INTRODUCTION}

One of the most intriguing questions in cognitive neuroscience today remains how conceptual information is represented in the brain. Embodied approaches to cognition suggest that conceptual information makes use of neural systems supporting actual perception, action, and emotion (Barsalou, 2008). In other words, concepts related to actions (such as action words, like grasp or run, or items used regularly in an action context, such as tools) are suggested to draw on the resources of neural motor areas, whereas concepts related to vision (such as color words or shapes) rely more heavily on visual cortex (Hauk, Davis, Kherif, \& Pulvermüller, 2008). Indeed, there is ample evidence from neuropsychological patient studies indicating that action-related concepts can be characterized as a unique conceptual category (Arévalo et al., 2007) and evidence from neuroimaging studies indicating that this conceptual category draws on the resources of the neural motor system (Rueschemeyer, Brass, \& Friederici, 2007; Tettamanti et al., 2005; Hauk, Johnsrude, \& Pulvermueller, 2004).

In particular, action-related objects (i.e., actual tools and artifacts) appear to selectively activate a network of neural areas including the ventral premotor cortex (vPMC), the inferior parietal cortex, the posterior lateral temporal cortex,

\footnotetext{
${ }^{1}$ Donders Centre for Cognition, Nijmegen, The Netherlands, ${ }^{2}$ Donders Centre for Cognitive Neuroimaging, Nijmegen, The Netherlands
}

gated whether a distinction can be made between words denoting (1) objects that can be picked up to move (e.g., volumetrically manipulable objects: bookend, clock) and (2) objects that must be picked up to use (e.g., functionally manipulable objects: cup, pen). The results show that functionally manipulable words elicit greater levels of activation in the fronto-parietal sensorimotor areas than volumetrically manipulable words. This suggests that indeed a distinction can be made between different types of manipulable objects. Specifically, how an object is used functionally rather than whether an object can be displaced with the hand is reflected in semantic representations in the brain.

and the medial-temporal cortex (Beauchamp \& Martin, 2007; Chao \& Martin, 2000; but see also Assmus, Giessing, Weiss, \& Fink, 2007). Different areas within this larger set have been ascribed different functional significance: The vPMC and the inferior parietal cortex have been described as a fronto-parietal network underlying functional action information about manipulable objects (i.e., reflecting knowledge about how a hammer is used), whereas posterior temporal areas are suggested to support information about nonbiological motion associated with manipulable objects as well as other visually encoded semantic properties of objects (Beauchamp \& Martin, 2007; Martin, 2007; Kable, Kan, Wilson, Thompson-Schill, \& Chatterjee, 2005; Kellenbach, Brett, \& Patterson, 2003). In particular, frontoparietal areas are seen to be active when participants make decisions about how to use manipulable objects in contrast to making decisions about what manipulable objects are used for (i.e., distinction between object manipulation vs. object function; see Canessa et al., 2008; Boronat et al., 2005; Kellenbach et al., 2003) or visual properties of manipulable objects (Ebisch et al., 2007).

Conceptual information about manipulable objects has commonly been investigated using picture stimuli; however, several studies have demonstrated that language stimuli can also be used to tap conceptual representations in the brain (Arévalo et al., 2007; Rueschemeyer et al., 2007; Boronat et al., 2005; Tettamanti et al., 2005; Hauk et al., 2004; Chao, Haxby, \& Martin, 1999; Grabowski, Damasio, \& Damasio, 1998; Grafton, Fadiga, Arbib, \& Rizzolatti, 1997). 
Chao et al. (1999) presented participants with both words and pictures of tools and found overlapping patterns of activation for both types of stimuli in temporal cortex. Hauk et al. (2004) presented participants with words denoting various actions (e.g., kick, pick) and showed a somatotopically organized pattern of activation in premotor cortex for words denoting actions carried out with different effectors. Saccuman et al. (2006) presented participants with picture stimuli and instructed participants to name the presented objects. Participants showed increased activation for production of nouns denoting manipulable objects (i.e., hammer) in contrast to nouns denoting nonmanipulable objects (i.e., traffic light) in vPMC and inferior parietal cortex. Thus, words as well as picture stimuli serve to access conceptual information in the brain, and for manipulable objects, conceptual information appears to be grounded in a fronto-parietal network and posterior temporal cortex.

In these previous studies, however, manipulability is not a well-defined parameter. Indeed, a number of recent studies have demonstrated that a distinction can be drawn between different types of manipulability (Bub, Masson, \& Cree, 2008; Masson, Bub, \& Newton-Taylor, 2008; Buxbaum, Kyle, Tang, \& Detre, 2006). Specifically, it has been shown that participants have very fast and automatic access to information about how an object is used with the hand (e.g., finger poking motion for pressing calculator buttons) and somewhat slower and less reliable access to information about how an object is displaced with the hand (e.g., how one would pick up the calculator to move it from desk to shelf). In previous neuroimaging studies examining brain activation patterns associated with manipulable objects, such a distinction between functional manipulation and nonfunctional manipulation (i.e., what Bub and Masson refer to as volumetric manipulation) has not been controlled for. In other words, manipulable object stimuli in previous studies were manipulable both in the sense that they could be lifted with the hand and in the sense that they required functional manipulation to use (as in the case of tools). Furthermore, manipulable items in previous studies have generally been contrasted with items too large or heavy to be held in the hand (e.g., traffic light or house). In other words, in addition to being perceptually quite different from manipulable objects in terms of size, nonmanipulable items are neither functionally manipulable (FM) nor volumetrically manipulable (VM), meaning that results of these studies could be attributed to either type of manipulability.

Conceptually, sensorimotor representations for tools and manipulable objects should reflect knowledge about how objects are used (i.e., functional manipulation) and not necessarily how they are moved (i.e., volumetric manipulation). In the current study, we therefore investigated whether words denoting objects associated with different types of manipulability (i.e., functional manipulability and volumetric manipulability) elicit different patterns of activation in sensorimotor areas. To this end, we presented participants in the scanner with words denoting manipulable objects, half of which were FM (i.e., they required manipulation for use, such as cup or hammer) and half of which allowed for volumetric manipulation but did not require manipulation for function (i.e., VM: they can be held in the hand but function without regular manipulation, such as clock or bookend). We hypothesized more activation for FM than for VM words in those brain areas involved in actual object manipulation, that is, fronto-parietal sensorimotor areas. The implication of this finding would be that functional manipulation or manipulation for use is reflected in the neural representation of object words.

\section{METHODS \\ Participants}

Fifteen students of the Radboud University participated in the study, all of whom were right-handed women between 18 and 25 years of age $(M=21, S D=2)$. All participants had normal or corrected-to-normal vision and no history of neurological disorders. Beforehand, all participants were informed about the experimental procedures, were given practice trials, and signed informed consent. Afterward, all students were awarded $€ 10$ for participating.

\section{Stimuli}

A total of 100 linguistic stimuli (i.e., letter strings comprising words and pseudowords) were created for the experiment. Eighty of the total 100 stimuli were real Dutch words and comprised the critical experimental stimuli; the remaining 20 stimuli were Dutch pseudowords (i.e., phonotactically and orthographically legal letter strings with no meaning in Dutch) and served as filler items and catch trials (see procedures below). The 80 critical word stimuli were matched for word length, frequency, and imageability. Critical stimuli belonged to one of two experimental conditions: (1) FM object condition or (2) VM object condition. Although all denoted objects were manipulable in the sense that they could be held in the hand or moved from one position to another, only FM objects require consistent manipulation for use. FM items are thus functionally and VM (e.g., cup, hammer), whereas VM objects are only VM (e.g., bookend, clock).

To test that stimuli were truly matched with regards to their volumetric manipulability (i.e., the ability of participants to lift the objects), a questionnaire was administered to 11 native Dutch speakers who did not participate in the subsequent fMRI experiment. In this questionnaire, participants were asked to rate words on a 7-point scale with respect to (1) their knowledge of the word ( $1=$ unknown, 7 = well known), (2) their familiarity with the word $(1=$ unfamiliar, 7 = very familiar), (3) their ability to image the object denoted by the word $(1=$ not imageable, $7=$ 
highly imageable), (4) whether they associated the object denoted by the word with an action $(-3=$ no action association, +3 = high action association), and (5) whether they could hold the object denoted by the word in their hands $(-3=$ cannot bold in hand, $+3=$ can bold in hand). The results of the questionnaire showed that objects were matched across conditions with respect to participants' knowledge (FM: $M=5.89$; VM: $M=5.88$ ), $|t(78)|<1$, ability to image denoted objects (FM: $M=$ 5.66; VM: $M=5.53),|t(78)|<1$, and frequency of word use (FM: $M=2.38$; VM: $M=2.15),|t(78)|<1$. Furthermore, participants agreed significantly that FM words were associated with an action $(M=0.62, S E=0.12), t(39)=$ $5.02, p<.001$, and were manipulable in the sense that they could be held in one's hand $(M=2.22, S E=$ $0.19), t(39)=11.60, p<.001$. For VM words, however, participants disagreed significantly that words were associated with an action $(M=-1.43, S E=0.12), t(39)=$ $-11.30, p<.001$, but agreed significantly that objects were nevertheless manipulable in the sense that they could be held in one's hand $(M=1.55, S E=0.24), t(39)=6.41$, $p<.001$.

Thus, stimuli were matched for relevant linguistic parameters, such as length, familiarity, imageability, and frequency. Crucially, participants reported that FM words but not VM words required manipulation for use but that both FM and VM words denoted objects that can be manipulated in the sense that they can be handheld.

\section{Procedure}

Participants were presented with a total of 100 experimental stimuli in the scanner. Stimuli belonged to one of three conditions: (1) words denoting FM objects, (2) words denoting VM objects, and (3) pseudowords (P). The 100 experimental stimuli comprised 80 critical items (i.e., $40 \mathrm{FM}$ words and $40 \mathrm{VM}$ words) and 20 catch trials (i.e., P words, see below). The order of stimulus presentation was randomized individually for each participant. All participants saw all experimental stimuli.

A trial consisted of visual presentation of a single word stimulus (or in the case of Null trials, presentation of a blank screen). At the beginning of each trial, a variable jitter time of $0,500,1000$, or $1500 \mathrm{msec}$ was included to improve the sampling rate of the BOLD signal. Following the jitter, a white fixation cross appeared on the screen for 300 msec. Directly following the fixation cross, the stimulus word was presented in the center of the screen for 2000 msec or until a response was recorded. Hereafter, a variable intertrial interval filled the remaining time, so that every trial lasted exactly $8000 \mathrm{msec}$.

Participants were instructed to read all words carefully and to perform a go/no-go lexical decision task, in which go responses should be made only in the $\mathrm{P}$ condition. For P words, participants were instructed to respond as quickly as possible. In this manner, we ensured that participants semantically processed all words (i.e., partici- pants had to comprehend the words to decide not to answer), but critical experimental stimuli were kept free of motor execution artifacts.

\section{fMRI Data Acquisition}

Functional images were acquired on a Siemens TRIO 3.0 T MRI system (Siemens, Erlangen, Germany) equipped with EPI capabilities, using a birdcage head coil for radio-frequency transmission and signal reception. BOLD-sensitive functional images were acquired using a single-shot gradient EPI sequence (echo time/repetition time $=30 / 2000 \mathrm{msec}$; 31 axial slices in ascending order, voxel size $=3.5 \times 3.5 \times$ 3.5). High-resolution anatomical images were acquired using an MPRAGE sequence (echo time $=3.03$; voxel size $=$ $1 \times 1 \times 1 \mathrm{~mm}, 192$ sagittal slices, field of view $=256$ ).

\section{fMRI Data Analysis}

Functional data were preprocessed and analyzed with SPM5 (Statistical Parametric Mapping, www.fil.ion.ucl.ac. $\mathrm{uk} / \mathrm{spm}$ ). Preprocessing involved removing the first three volumes to allow for T1 equilibration effects. Rigid body registration along three translations and three rotations was applied to correct for small head movements. Subsequently, the time series for each voxel was realigned temporally to acquisition of the middle slice (Slice 17) to correct for slice timing acquisition delays. Images were normalized to a standard EPI template centered in Montreal Neurological Institute space and resampled at an isotropic voxel size of $2 \mathrm{~mm}$. Low-frequency signal changes and baseline drifts were removed by applying a temporal high-pass filter to remove frequencies lower than $1 / 120 \mathrm{~Hz}$. The normalized images were smoothed with an isotropic 10-mm FWHM Gaussian kernel. The ensuing preprocessed fMRI time series were analyzed on a subject-by-subject basis using an event-related approach in the context of the general linear model with regressors for each condition (FM, VM, P, and Null) convolved with a canonical hemodynamic response function. The parameters from the motion correction algorithm were included in the model as effects of no interest.

For each participant, four contrast images were generated, representing the main effect of reading words belonging to each category versus a resting baseline (FM-baseline, VM-baseline) as well as the main effects of object manipulability (FM objects-VM objects and VM-FM). Because individual functional data sets had been aligned to the standard stereotactic reference space, a group analysis based on the contrast images could be performed. Single-participant contrast images were entered into a second-level random effects analysis for the critical contrast of interest. The group analysis consisted of a one-sample $t$ test across the contrast images of all subjects that indicated whether observed differences between conditions were significantly distinct from zero. To protect against false-positive activation, a double threshold was applied, by which only voxels 
with a $p<.005$, uncorrected, and a volume exceeding 300 voxels were considered (Forman et al., 1995).

\section{RESULTS}

\section{Behavioral Results}

The results of the behavioral data show that participants were alert and performing the lexical decision task (performance rates: $\mathrm{FM}, M=98.5 \%, S E=0.43$; VM, $M=98.39 \%$, $S E=0.42)$. One participant, who made over $50 \%$ errors, was excluded from the data analysis. Thus, results of 14 participants entered the analysis.

\section{Neuroimaging Results}

A list of significant activations can be seen in Table 1 and Figures 1 and 2 .

The two baseline contrasts (i.e., FM-baseline and VMbaseline) revealed largely overlapping areas for each word reading condition (see Figure 1). Large activations were observed in both hemispheres extending from the posterior insula into lateral inferior parietal cortex (inferior postcentral gyrus), across the sylvian fissure into the mid to anterior reaches of the superior temporal gyrus. In addition, both contrasts revealed significantly increased activation in the cuneus, extending toward the fusiform gyrus. Both contrasts also showed increased activation for the word conditions in the dorso-lateral pFC anterior to the precentral sulcus and superior to the inferior frontal sulcus. In addition, the FM-baseline condition yielded significant results in the medial frontal cortex within both the supplementary motor and the pre-SMAs. This activation was not observed in the VM-baseline condition.

Whole-brain analysis revealed two areas to be more strongly activated in response to FM as compared with non-FM object words $(\mathrm{FM}>\mathrm{VM})$. These were the pre-SMA and the

Table 1. Brain Regions Showing Significantly More Activation for FM Than for VM Objects ( $p<.005, k>300$ voxels)

\begin{tabular}{|c|c|c|c|c|c|}
\hline Brain Region & $Z_{\max }$ & $\begin{array}{c}\text { Extent } \\
\text { (Voxels) }\end{array}$ & $x$ & $y$ & $z$ \\
\hline Pre-SMA & & 395 & & & \\
\hline $\begin{array}{l}\text { Right medial superior } \\
\text { frontal gyrus }\end{array}$ & 3.94 & & 4 & 18 & 52 \\
\hline $\begin{array}{l}\text { Left medial superior } \\
\text { frontal gyrus }\end{array}$ & 2.94 & & -15 & 2 & 52 \\
\hline Left inferior parietal cortex & & 615 & & & \\
\hline Left supramarginal gyrus & 3.35 & & -34 & -36 & 36 \\
\hline Left postcentral gyrus & 3.32 & & -56 & -30 & 28 \\
\hline Left inferior precentral gyrus & 3.30 & & -58 & -10 & 18 \\
\hline
\end{tabular}

The maximum $Z$ scores, the cluster extent (in voxels), and the Montreal Neurological Institute coordinates are reported. left inferior parietal lobule extending to inferior frontal cortex. The inferior fronto-parietal activation had three local maxima: (1) on the border between the inferior precentral gyrus (inferior bank of central sulcus), (2) in the inferior postcentral gyrus, and (3) in the supramarginal gyrus extending toward the intraparietal sulcus.

No areas were seen to be more active for words associated with nonfunctional versus functional manipulability $(\mathrm{VM}>\mathrm{FM})$.

\section{DISCUSSION}

In the current experiment, lexical-semantic representations of words belonging to two categories-(1) words denoting FM objects and (2) words denoting VM objects-were investigated. The critical contrasts of interest indicate that words denoting FM objects elicit greater activation than words denoting VM objects in several sensorimotor areas of the brain. In particular, activation was seen in the vPMC (inferior portion of precentral gyrus), in the inferior parietal cortex, and in the pre-SMA (see Figure 2). This indicates that the specific way in which an object is manipulated is reflected in the neural representation of object words.

In addition to the contrasts of interest, two baseline contrasts were calculated, testing effects of word recognition versus a resting baseline. Broad activations within frontotemporal language areas as well as activation in visual cortex in both of these contrasts are indeed consistent with previous literature on word reading (see Figure 1). These findings are not further discussed because the question of interest in this study was not centered on effects of visual word recognition.

Participants' responses on a questionnaire revealed that words in both conditions (i.e., FM and VM words) denote manipulable objects in the sense that all objects can be handheld. Thus, a general association between the hand effector and the object denoted was present for all words. Furthermore, all word stimuli could thus have been included in the set of "manipulable objectsh in previous studies. The questionnaire further revealed that FM words were associated with a specific action whereas VM words were not. This distinction was the critical point under investigation. Specifically, we hypothesized that FM words would show more activation in motor areas than VM words because FM objects must be manipulated to function whereas VM words can be manipulated but do not require manipulation for use. It should be noted here that action association and manipulability scores did correlate in the questionnaire responses, such that words more highly associated with specific actions were also judged to be more manipulable. This is intuitively plausible because objects that must be manipulated for use will undoubtedly have been taken in the hand more frequently over the course of a participant's life. However, the critical point in this study is that both FM and VM words are seen to be manipulable (as indicated by the fact that responses to both word categories differ significantly from 
Figure 1. Differences in BOLD response for both word categories in contrast to a resting baseline $(p<.001$, $k>300)$. Areas showing significantly greater activation for FM object words are depicted in red; areas showing greater activation for VM objects words are depicted in blue; overlapping FM + VM activations are shown in pink. In addition, percent signal change for FM and VM words compared with resting baseline

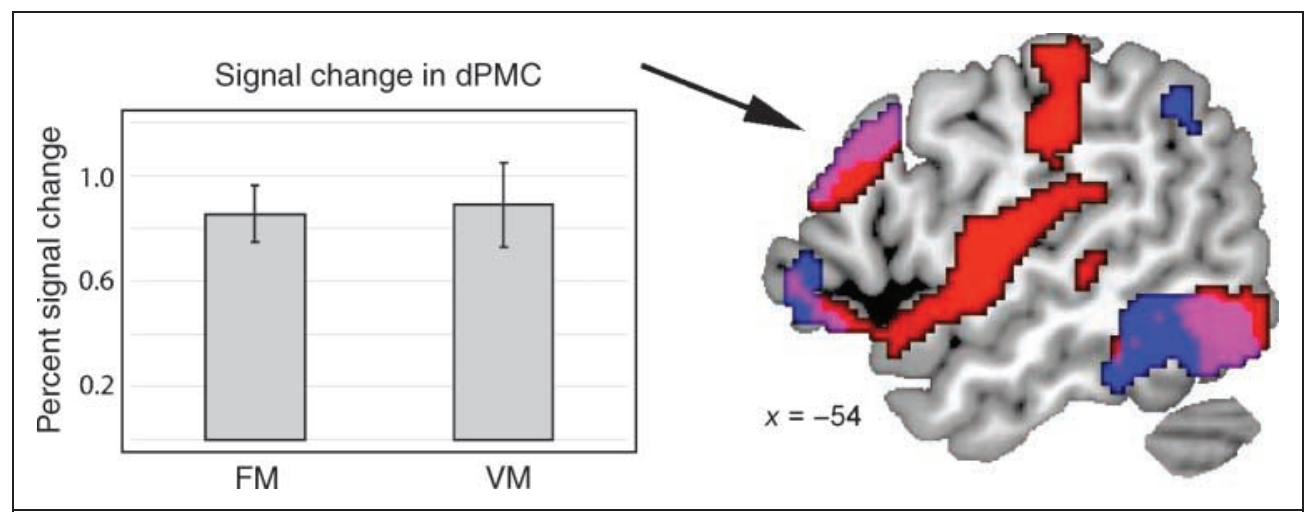

in the dorsal premotor cortex (dPMC: $-44,24,40$ ) is shown in the bar diagram. In the APMC, FM and VM words both elicit activation greater than zero; however, this activation does not differ between the word conditions.

zero), which distinguishes the current stimulus set from previous studies.

One further critical point with regards to the questionnaire is that participants did report confusion about what was meant by the question about specific action associations. In particular, although participants were quite clear about when an object was not associated with a specific action, they found it difficult to determine whether objects were associated with a specific action. Specifically, words associated with multiple actions (e.g., cup: filling the cup, bringing the cup to the mouth, drinking) were not clearly associated with one specific action for participants. Thus, responses to FM words were actually surprisingly low $(M=0.62)$, although nevertheless significantly different from zero.
Figure 2. Differences in BOLD response for words denoting FM versus VM $(\mathrm{FM}>\mathrm{VM})$ objects $(p<.005, k>300)$. Significant differences in activation are seen in the left inferior parietal lobule, extending from the supramarginal gyrus (SMG) across the inferior portion of the postcentral gyrus (L. postCG) and into the inferior portion of the precentral gyrus (L. preCG). In addition, significant modulation of BOLD response is seen in the pre-SMA. Percent signal change is shown for the area surrounding the peak voxel in each activated area.

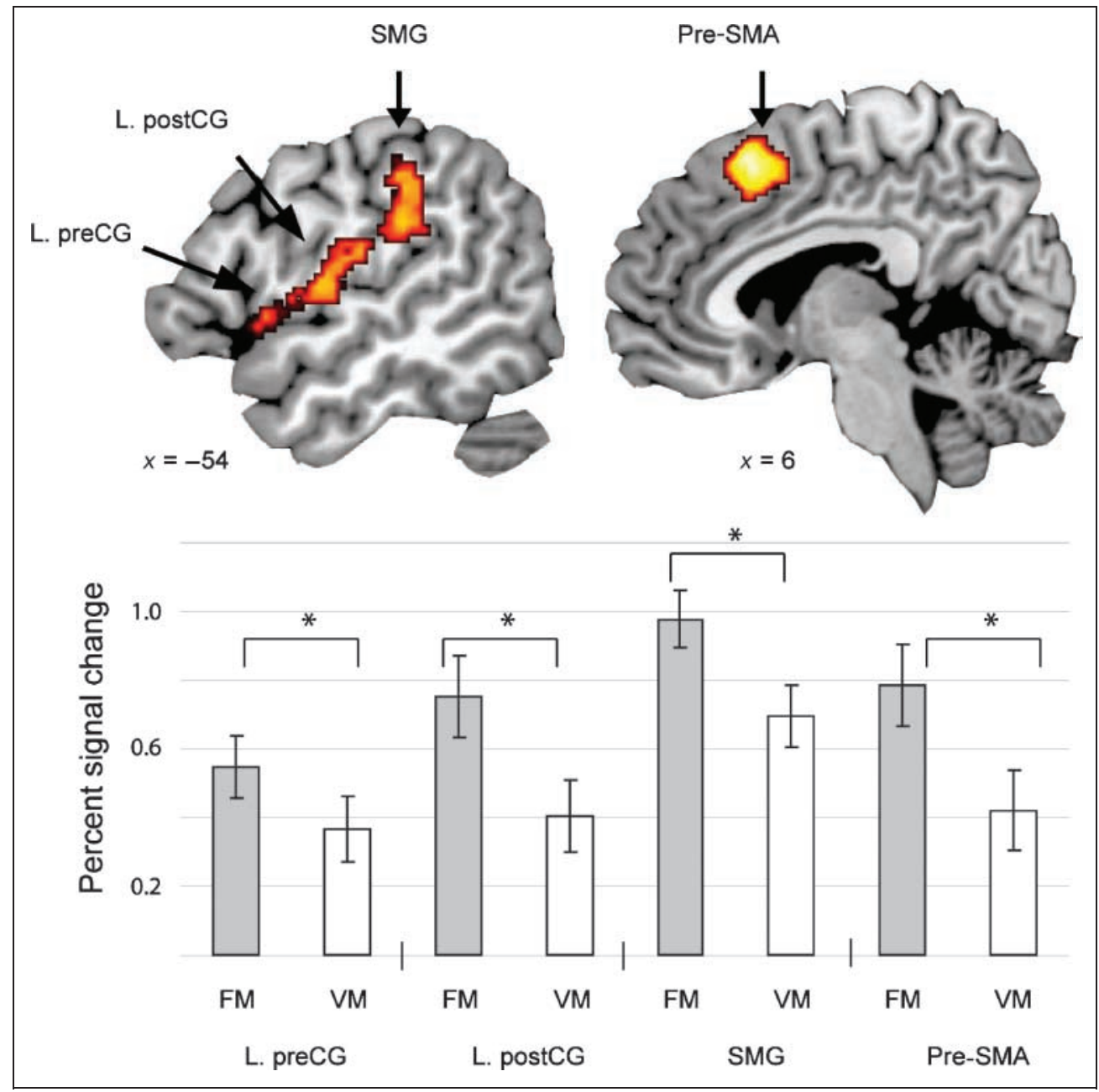


Previous research has shown that processing information about tools relies on several distinct cortical areas in the left hemisphere, including the anterior intraparietal sulcus in the inferior parietal lobule and the vPMC as well as selective areas in posterior temporal cortex (Beauchamp \& Martin, 2007). Fronto-parietal activations in response to tool presentation are suggested to underlie knowledge about how to use or manipulate a tool, whereas temporal activations are thought to support recognition of various visual characteristics of tools (i.e., form, visual motion) (Beauchamp \& Martin, 2007; Culham \& Valyear, 2006). In the current experiment, we investigated the processing of objects, all of which are tools in some sense but only half of which require a specific manipulation for use. Therefore, although all items had similar perceptual characteristics (i.e., all can be moved with the hand, all have been seen in motion, and all are of relatively small size), only one set of items was associated with a specific action. We expected differences in activation between the stimulus types primarily in brain areas associated with functional manipulation, that is, in the fronto-parietal network. This is precisely what was seen in the current study.

Interestingly, frontal activation in the current study is restricted to the vPMC and does not include dorsal premotor (dPM) areas reported in previous studies of hand movements and hand action word representations (Buccino et al., 2005; Tettamanti et al., 2005; Hauk et al., 2004). This may be due to the fact that, in contrast to the stimuli used in these previous studies, both FM and VM objects in our study denote generally manipulable objects. In other words, previous studies reporting extensive dPM activation contrasted words denoting manipulable objects and hand actions with words denoting nonmanipulable objects and actions irrelevant to the hand (e.g., in essence, cup vs. house or grasp vs. kick, respectively). The dPM cortex is thought to support execution and observation of general hand actions (Buccino et al., 2001; Yousry et al., 1997), and thus APM activations in these studies probably reflect information the participant has about how to grasp or touch manipulable objects. As indicated by the results of our stimulus questionnaire, all objects in the current study (i.e., both FM and VM objects) are manipulable in the sense that they can be grasped with the hand; therefore, extensive APM activation may be postulated for both word categories (see also Gerlach, Law, \& Paulson, 2002). Indeed, a baseline contrast (depicted in Figure 1) provides evidence that this is the case. Both FM and NM words show significant signal increase compared with a resting baseline. The difference between the two conditions is, however, not significant.

In addition to the anticipated fronto-parietal activation, greater activation was seen in the medial pFC, specifically in the pre-SMA. The precise function of pre-SMA remains a topic of controversy; however, because of its tight links to ventral premotor areas (in contrast to SMA proper), it is attributed a more cognitive role in the establishment and retrieval of motor sequences and visuomotor associa- tions (Picard \& Strick, 2001). In the current experiment, FM words are suggested to have stronger associations to a specific type of motor information than VM words. This link between a cognitive task (i.e., word processing) and a general motor association may be supported by preSMA (see also Postle, McMahon, Ashton, Meredith, \& de Zubicaray, 2008).

Thus, FM words show significant activation in areas underlying actual object manipulation. Previous behavioral studies have shown a distinction in how functional and volumetric manipulation parameters are processed with respect to single words. Specifically, whereas FM information is processed very quickly and possibly automatically (i.e., on the order of $250 \mathrm{msec}$ ), VM information appears to become activated only at a later stage (i.e., after $750 \mathrm{msec}$; see Masson et al., 2008). Masson et al. (2008) thus suggest that knowledge about an object's function is central to an object's meaning, whereas knowledge about an object's form is considered only when relevant (i.e., only when the participant prepares to displace the object). Several electrophysiological studies on the timing of action-word processing have also indicated that "action information" becomes available very early in word processing (150200 msec; Pulvermueller, Shtyrov, \& Ilmoniemi, 2005; Hauk \& Pulvermueller, 2004). The current study did not attempt to disentangle FM from VM information within single words, as the research cited above indicates that the temporal lag between processing of FM and VM sentences is too short to allow for a dissociation using fMRI. Instead, we investigated the neural representation of words denoting objects associated with both FM and VM parameters (e.g., cup, which must be brought to the mouth to function but which can clearly also be displaced) with words denoting objects with only a VM parameter (e.g., vase, which holds flowers with no regular manipulation necessary but which can clearly be held in the hand and moved). The results are in line with those of Masson et al. because a clear distinction between FM and VM information can be made. Specifically, the results demonstrate that words with an FM association elicit more activation than VM words in areas generally associated with sensorimotor processing.

It should be noted here that although we interpret this difference to reflect information about functional object use, it is of course also the case that FM objects are also simply more associated with action (as indicated also by the results of the questionnaire). Thus, results are in holding with previous studies showing more activation in action-related areas for words with stronger action associations (Hauk et al., 2008; Davis, Meunier, \& MarslenWilson, 2004). However, the behavioral work cited above also clearly shows that knowledge about functional and volumetric manipulations of objects can be dissociated, and the current results thus also reveal something about the neural substrates underlying this dissociation.

The results of the current study are in general agreement with the framework of embodied cognition, which 
posits that conceptual information makes use of neural systems supporting actual perception, action, and emotion. Specification of the time scale along which perception and action systems become involved in lexical-semantic processing remains an open question in embodied language cognition (see Barsalou, 2008; Barsalou, Santos, Simmons, \& Wilson, 2008; Pulvermueller, Shtyrov, et al., 2005). Furthermore, it remains unclear whether perceptual and action systems are necessarily involved in lexical-semantic processing or whether simulation reflects deeper, postlexical semantic processing. Empirical evidence for both perspectives can be found in the literature (e.g., Hoenig, Sim, Bochev, Herrnberger, \& Kiefer, 2008; Pulvermueller, Hauk, Nikulin, \& Ilmoniemi, 2005). The results presented here do not speak to these important issues, as neither the timing of simulation processes nor the flexibility of semantic representations is addressed by the current design. The current study does indicate that the real-world experiences one has had with an object (i.e., the way in which an object is manipulated) influence the lexical-semantic representation of the word form referring to that object. This finding is consistent with both embodied perspectives. Further research is needed to delve into how flexible these representations are within individuals. For example, we argue that the way in which an object is used functionally is critical in determining the neuroanatomical profile of its lexical-semantic symbol (i.e., the word denoting the object). For the population, we investigated that clocks belong to a category of items that are volumetrically but not FM (i.e., the clock is hung on the wall or placed on the bedside table but requires no regular further manipulation to work). For a population of clockmakers, the association might be entirely different. Thus, individual experience and expertise may certainly play a role in the lexical-semantic representations we observe in the current study.

\section{Conclusion}

In conclusion, the results of the current study show that different types of manipulability are reflected in object word representations. Specifically, representations of FM and VM are dissociable in the brain, and words denoting objects associated with FM or VM parameters reflect this dissociation. Although the neural representation of FM and VM words is largely overlapping, FM words show that additional activation increases in several classical sensorimotor brain areas, including the vPMC, the inferior parietal cortex, and the pre-SMA. The results show that how an object is typically manipulated is critical in determining how the semantic representation of the object is processed in the brain. This indicates that embodied semantic representations are quite specific in the type of experiential information they contain; however, future research is required to determine how automatic or necessary embodied representations are for language processing.

\section{Acknowledgments}

The present study was supported by a VENI Grant (016-094-053) to the first author and a VICI Grant to the last author (453-05-001) from the Dutch Organization for Scientific Research (NWO) and the ICIS project (BSIK03024) sponsored by the Dutch Ministry of economic affairs. The authors would further like to thank Paul Gaalman for technical support.

Reprint requests should be sent to Shirley-Ann Rueschemeyer, Donders Centre for Brain, Cognition and Behaviour, Centre for Cognition, Montessorilaan 3, 6525 HR, Nijmegen, The Netherlands, or via e-mail: s.rueschemeyer@donders.ru.nl.

\section{REFERENCES}

Arévalo, A., Perani, D., Cappa, S. F., Butler, A., Bates, E., \& Dronkers, N. (2007). Action and object processing in aphasia: From nouns and verbs to the effect of manipulability. Brain and Language, 100, 79-94.

Assmus, A., Giessing, C., Weiss, P., \& Fink, G. R. (2007). Functional interactions during the retrieval of conceptual action knowledge: An fMRI study. Journal of Cognitive Neuroscience, 19, 1004-1012.

Barsalou, L. (2008). Grounded cognition. Annual Review of Psychology, 59, 617-645.

Barsalou, L., Santos, A., Simmons, W., \& Wilson, C. (2008). Language and simulation in conceptual processing. In $\mathrm{M}$. De Vega, A. M. Glenberg, \& A. C. Graesser (Eds.), Symbols, embodiment, and meaning (pp. 245-283). Oxford: Oxford University Press.

Beauchamp, M., \& Martin, A. (2007). Grounding object concepts in perception and action: Evidence from fMRI studies of tools. Cortex, 43, 461-468.

Boronat, C., Buxbaum, L., Coslett, H. B., Tang, K., Saffran, E., Kimberg, D., et al. (2005). Distinctions between manipulation and function knowledge of objects: Evidence from functional magnetic resonance imaging. Cognitive Brain Research, 23, 361-373.

Bub, D., Masson, M., \& Cree, G. (2008). Evocation of functional and volumetric gestural knowledge by objects and words. Cognition, 106, 27-58.

Buccino, G., Binkofski, F., Fink, G. R., Fadiga, L., Fogassi, L., Gallese, V., et al. (2001). Action observation activates premotor and parietal areas in a somatotopic manner: An fMRI study. European Journal of Neuroscience, 13, 400-404.

Buccino, G., Riggio, L., Melli, G., Binkofski, F., Gallese, B., \& Rizzolatti, G. (2005). Listening to action-related sentences modulates the activity of the motor system: A combined TMS and behavioral study. Cognitive Brain Research, 24, 355-363.

Buxbaum, L., Kyle, K., Tang, K., \& Detre, J. (2006). Neural substrates of knowledge of hand postures for object grasping and functional object use: Evidence from fMRI. Brain Research, 1117, 175-185.

Canessa, N., Borgo, F., Cappa, S. F., Perani, D., Falini, A., Buccino, G., et al. (2008). The different neural correlates of action and functional knowledge in semantic memory: An fMRI study. Cerebral Cortex, 18, 740-751.

Chao, L., \& Martin, A. (2000). Representation of manipulable man-made objects in the dorsal stream. Neuroimage, 12, 478-484.

Chao, L. L., Haxby, J. V., \& Martin, A. (1999). Attribute-based neural substrates in temporal cortex for perceiving and knowing about objects. Nature Neuroscience, 2, 913-919.

Culham, J. C., \& Valyear, K. (2006). Human parietal cortex in action. Current Opinion in Neurobiology, 16, 205-212.

Davis, M., Meunier, F., \& Marslen-Wilson, W. (2004). Neural responses to morphological, syntactic, and semantic 
properties of single words: An fMRI study. Brain and Language, 89, 439-449.

Ebisch, S., Babiloni, C., Del Gratta, C., Ferretti, A., Perrucci, M., Caulo, M., et al. (2007). Human neural systems for conceptual knowledge of proper object use: A functional magnetic resonance imaging study. Cerebral Cortex, 17, 2744-2751.

Forman, S., Cohen, J., Fitzgerald, M., Eddy, W., Mintun, M., \& Noll, D. (1995). Improved assessment of significant activation in functional magnetic resonance imaging (fMRI): Use of a cluster-size threshold. MRM, 33, 636-647.

Gerlach, C., Law, I., \& Paulson, O. (2002). When action turns into words. Activation of motor-based knowledge during categorization of manipulable objects. Journal of Cognitive Neuroscience, 14, 1230-1239.

Grabowski, T., Damasio, H., \& Damasio, A. (1998). Premotor and prefrontal correlates of category-related lexical retrieval. Neuroimage, 7, 232-243.

Grafton, S., Fadiga, L., Arbib, M., \& Rizzolatti, G. (1997). Premotor cortex activation during observation and naming of familiar tools. Neuroimage, 6, 231-236.

Hauk, O., Davis, M., Kherif, F., \& Pulvermüller, F. (2008) Imagery or meaning? Evidence for a semantic origin of category-specific brain activity in metabolic imaging. European Journal of Neuroscience, 27, 1856-1866.

Hauk, O., Johnsrude, I., \& Pulvermueller, F. (2004). Somatotopic representation of action words in human motor and premotor cortex. Neuron, 41, 301-307.

Hauk, O., \& Pulvermueller, F. (2004). Neurophysiological distinction of action words in the fronto-central cortex. Human Brain Mapping, 21, 191-201.

Hoenig, K., Sim, E., Bochev, V., Herrnberger, B., \& Kiefer, M. (2008). Conceptual flexibility in the human brain: Dynamic recruitment of semantic maps from visual, motor, and motion-related areas. Journal of Cognitive Neuroscience, 20, 1799-1814.

Kable, J., Kan, I., Wilson, A., Thompson-Schill, S., \& Chatterjee, A. (2005). Conceptual representations of action in the lateral temporal cortex. Journal of Cognitive Neuroscience, 17, $1855-1870$.
Kellenbach, M., Brett, M., \& Patterson, K. (2003). Actions speak louder than functions: The importance of manipulability and action in tool representation. Journal of Cognitive Neuroscience, 15, 30-46.

Martin, A. (2007). The representation of object concepts in the brain. Annual Review of Psychology, 58, 25-45.

Masson, M., Bub, D., \& Newton-Taylor, M. (2008). Language-based access to gestural components of conceptual knowledge. Quarterly Journal of Experimental Psychology, 61, 869-882.

Picard, N., \& Strick, P. (2001). Imaging the premotor areas. Current Opinion in Neurobiology, 11, 663-672.

Postle, N., McMahon, K., Ashton, R., Meredith, M., \& de Zubicaray, G. (2008). Action word meaning representations in cytoarchitectonically defined primary and premotor cortices. Neuroimage, 43, 634-644.

Pulvermueller, F., Hauk, O., Nikulin, V., \& Ilmoniemi, R. (2005). Functional links between motor and language systems. European Journal of Neuroscience, 21, 793-797.

Pulvermueller, F., Shtyrov, Y., \& Ilmoniemi, R. (2005). Brain signatures of meaning access in action word recognition. Journal of Cognitive Neuroscience, 17, 884-892.

Rueschemeyer, S.-A., Brass, M., \& Friederici, A. D. (2007). Comprehending prehending: Neural correlates of processing verbs with motor stems. Journal of Cognitive Neuroscience, 19, 855-865.

Saccuman, C., Cappa, S., Bates, E., Arevalo, A., Della Rosa, P., Danna, M., et al. (2006). The impact of semantic reference on word class: An fMRI study of action and object naming. Neuroimage, 32, 1865-1878.

Tettamanti, M., Buccino, G., Saccuman, M., Gallese, V., Danna, M., Scifo, P., et al. (2005). Listening to action-related sentences activates fronto parietal motor circuits. Journal of Cognitive Neuroscience, 17, 273-281.

Yousry, T., Schmid, U., Alkadhi, H., Schmidt, D., Peraud, A., Buettner, A., et al. (1997). Localization of the motor hand area to a knob on the precentral gyrus. Brain, 120, $141-157$. 